\title{
Présentation de résumés de recherche Assemblée scientifique annuelle de l'ACMU Du 26 au 29 avril 2004
}

\section{Appel de résumés de recherche}

L'ACMU invite tous les chercheurs à soumettre leur recherche clinique ou de base originale pour présentation lors de l'Assemblée scientifique de l'ACMU 2004. Le Comité organisateur a travaillé très fort pour s'assurer que la présentation des travaux de recherche soit bien en évidence lors de cette assemblée. Les résumés doivent représenter une recherche originale qui

a) n'a pas été publiée ni présentée lors d'une assemblée scientifique nationale (canadienne) et

b) n'a pas déjà été publiée dans un journal ou une revue ou un livre en ligne.

Tous les résumés doivent nous parvenir en format électronique au plus tard le 20 janvier 2004.

\section{Instructions pour la préparation des résumés}

Veuillez suivre les instructions. Le non respect de ces instructions entraînera la disqualification de votre résumé. Les résumés peuvent être soumis en français ou en anglais. Ils doivent être tapés à simple interligne, de façon claire et lisible. Ils doivent être soumis électroniquement de la manière suivante : visitez le site web du Consortium de recherche de l'ACMU (www.caep.ca/rc). Cliquez sur Call for CAEP Abstracts et suivez les instructions. Remplissez les cases obligatoires. Ne laissez aucun champ non rempli. Les résumés doivent comprendre dans l'ordre : Introduction, Méthodes, Résultats, Conclusions, et chaque titre doit être souligné. Donnez l'affiliation hospitalière, la ville et la province de résidence de l'auteur principal seulement. Le texte du résumé doit être justifié à gauche et comporter un maximum de 300 mots. Les abréviations doivent être gardées à un strict minimum et être définies lors de leur première utilisation dans le texte. Les graphiques et les tableaux doivent pouvoir s'insérer dans le cadre prévu pour le résumé.

\section{Processus d'étude des résumés}

Tous les résumés seront étudiés par au moins trois réviseurs de l'ACMU à l'aide d'une grille d'évaluation normalisée. Les réviseurs du Comité de recherche ne connaîtront pas le(s) nom(s) de(s) auteur(s) ni leur affiliation hospitalière. Les réviseurs n'évalueront pas de résumés provenant de leur propre province. Des déclarations de conflit d'intérêt seront fournies à chacun des réviseurs. Les résumés seront choisis pour une présentation orale ou sur affiche. Pour les présentations orales, la longueur sera de 10 minutes, 5 minutes étant prévues pour la discussion. Les affiches seront installées dans un endroit central du centre des congrès. La notification d'acceptation sera transmise par courriel ou par télécopieur au plus tard le $\mathbf{1}^{\text {er }}$ mars 2004. Par conséquent, toutes les coordonnées personnelles doivent être exactes lors de la demande. Nous regrettons de ne pouvoir donner de notification par téléphone.

Les horaires complets de présentation seront affichés sur le site web de l'ACMU (www.caep.ca) et sur celui du Consortium de recherche (www.caep.ca/rc). La publication du résumé original dans le JCMU se fera une fois que les résumés auront été acceptés.

\section{Concours de présentation de résumés de recherche des résidents}

Fujisawa Canada a accepté une fois de plus d'appuyer le Concours de recherche des résidents. Le Comité de recherche de l'ACMU assumera les frais de déplacement de dix résidents dont les résumés auront été choisis. Les résidents doivent faire partie de programmes canadiens de MU agrés (CMFC ou RCPS) et doivent avoir obtenu leur diplôme au cours de l'année précédente (i.e. juillet 2003). Le résident doit être le chercheur principal et être responsable du développement du projet, être celui qui présente le résumé (présentation orale) et être l'auteur nommé en premier. Les fellows (médecine d'urgence pédiatrique et autres) ne sont pas admissibles à ce concours, à moins que leur recherche n'ait été terminée pendant leur résidence en MU.

\section{Concours de résumés de recherche pour les étudiants en médecine}

Le Comité de recherche de l'ACMU choisira les résumés de cinq étudiants en médecine pour ce prix. La recherche doit avoir eu lieu dans un établissement canadien et l'étudiant doit être le chercheur principal et la personne responsable du développement du projet. Il doit également être celui qui présente le résumé (présentation orale) et être l'auteur nommé en premier. 